$4^{\text {th }}$ International Conference on Biotechnology Applications in Agriculture (ICBAA), Benha University, Moshtohor and Hurghada, 4-7 April 2018, Egypt

\title{
Genomic approaches and bioinformatics tools to identify genomic regions for economic traits and their applications in chicken breeding programs
}

\author{
MOSTAFA K. NASSAR \\ Department of Animal Production, Faculty of Agriculture, Cairo University, 12613 Giza, Egypt \\ Corresponding author: mostafa.nassar@agr.cu.edu.eg
}

\begin{abstract}
Most of the economic traits considered in genetic improvement programs are of quantitative nature. They are genetically determined by many genes. To apply major genes or linked markers in gene- or marker-assisted selection program, they must first be identified in the genome. Mapping genomic regions for the economic traits is the first step to identify genes influencing traits of interest. This report gives the most comprehensive information on discovering quantitative traits loci (QTL) and their underlying genes for economic traits in chicken, in particular on chromosome 4 (GGA4), using up-to-date genomic approaches and bioinformatics tools. This work is based on several publications (Goraga et al. 2010, 2012; Nassar and Brockmann 2011, 2013; Nassar et al. 2012, 2013, 2015; Lyu et al. 2016, 2017) and other published QTL results in chicken QTL database (Chicken QTLdb). I had done this work in collaboration with Humboldt-Universität zu Berlin, Germany, during the year 2010 to 2017. In brief, we mapped several genomic regions on 22 chromosomes affecting 24 traits. The majority of identified loci showed additive effects on several growth and body composition traits. The biggest effect on analysed traits was detected on the distal region of GGA4. The confidence interval of the QTL region on GGA4 harbours hundreds of genes. The final identification of genes and mutations will contribute to our understanding of the complex inheritance pattern of growth regulation, muscle development and fat deposition in chicken. Such information would support breeders in using this information for genetic improvement in breeding programs.
\end{abstract}

Keywords: growth, muscle mass, fat deposition, candidate gene, QTL mapping, bioinformatics, whole genome sequencing, microsatellite, SNP arrays, linkage and association analyses, molecular breeding

\section{Background}

One of the main concerns of the livestock industry is to improve economically important traits in animals. Growth traits and in particular the yield of muscle mass and the amount of white adipose tissue deposited in the body are the most important economical traits that influence the nutritional and economic values of chickens. These phenotypes are of complex nature. They are genetically determined by expression of more than one gene. Despite rapid developments in genetics, genomics and bioinformatics tools, fine dissection of complex traits has remained challenging task in livestock. Knowledge of quantitative trait loci (QTL) and their underlying genes (QTG) contributing to these traits, which cannot be measured directly on living chickens, would support breeders in using this information for genetic improvement in breeding programs. QTL are often found on different chromosomes. Sometimes a cluster of closely linked polymorphic genes within a QTL region is responsible for the quantitative variation of a trait (Abiola et al. 2003: Members of the Complex Trait Consortium 2003).

The design of mapping population is the crucial step in genome-wide QTL scan. A powerful approach for mapping QTL is to use crosses between two populations that differ (high vs. low) in the traits of interest
(Andersson 2001). This means, different QTL alleles are completely fixed with parental lines and segregate in the crossbred $F_{2}$ populations. Thus, in an $F_{2}$ population the additive and dominance QTL effects can be estimated (Alfonso and Haley 1998). As a first step in gene discovery, usually a linkage or association study is performed that maps genomic loci (QTL) affecting the expression of complex traits and which contains hundreds of genes. In a subsequent step, these QTL are fine mapped in such strategies to identify the one or several causative genes.

Different crosses between diverse chicken breeds or within breeds have been used to map genomic loci affecting growth performance and carcass composition traits. For instance, crosses between high-and lowgrowth selected lines (Jennen et al. 2004; Jacobsson et al. 2005; Park et al. 2006; Nadaf et al. 2009) and crosses between phenotypically and genetically different breeds, e.g. White Leghorn (layer) with either broiler (meat-type chicken) (Sewalem et al. 2002; Carlborg et al. 2004; Ikeobi et al. 2004; Schreiweis et al. 2005; Zhou et al. 2006a, b), Rhode Island Red (Tuiskula-Haavisto et al. 2002), red junglefowl (Kerje et al. 2003), Oh-Shamo (Tsudzuki et al. 2007), or Golden Montazah (Abdel Alal et al. 2016; Khalil et al. 2016) were produced. Moreover, crosses between broiler and Fayoumi (Zhou et al. 2006a, b) or layer lines (Ambo et al. 2009; Campos et al. 2009) have been used for QTL mapping. Because 
different chicken strains or lines can harbour different variants of genes that control traits of interest, additional mapping studies can help to add genetic variation contributing to our traits of interest and they can be used for combined analysis from other crosses to fine map major QTL effects. Therefore, the aims of this project were: 1) to identify QTL affecting growth performance, muscle mass, carcass parts and fat deposition, and 2) to fine map and identify potential genes for a major growth QTL on chromosome 4 (GGA4) in chicken. The final identification of genes and their mutations will contribute to our understanding of the complex inheritance pattern of growth regulation, muscle development and fat deposition in chicken. Such information could be more emphasized in animal breeding programs using marker-assisted selection (MAS) or genomic selection (GS) in order to increase the selection response in a reasonable time frame.

\section{Material and Strategies}

Animals: An $\mathrm{F}_{2}$ mapping population and advanced intercross line (AIL; $F_{10}$ to $F_{12}$ ) has been established from the initial crosses between the two different lines New Hampshire (NHI) and White Leghorn (WL77), in which we used about 1000 animals. The lines NHI and WL77 had been selected for high body weight at the age of 20 weeks and for low egg weight during laying period, respectively. Afterwards, the lines were inbred. NHI chickens show a two-fold higher body weight at selection age compared to WL77 (Goraga et al. 2010).

Phenotypes: 24 phenotypes of growth performance and body composition were used in this study. Growth performance included body weight and body weight gain at different developmental stages between hatch and 20 weeks. Body composition measurements included weights of carcass, breast muscle, wings, residual carcass, drumsticks and thighs, as well as visceral and subcutaneous adipose tissue masses and intramuscular fat content at the age of 20 weeks.

Genotyping and sequencing: Genome-wide linkage analysis using 123 microsatellite (short sequence repeats; SSR) and single nucleotide polymorphism (SNP) loci were sued to map QTL covering 25 chromosomes (for detail see Goraga et al. 2012; Nassar et al. 2012, 2013, 2015). Chicken 60K and 600K SNP chips as well as whole genome sequencing were used to fine map and characterize the major growth QTL in the distal part of chromosome 4 (for detail see Lyu et al. 2017).

Analyses and bioinformatics tools: The program GridQTL (http://www.gridqtl.org.uk/) was used for QTL analysis. Sex-averaged genetic map (Kosambi) was used for the QTL scans. Interval mapping was performed using multiple regressions (Nassar et al. 2012, 2013, 2015). Once a single QTL in a linkage group was identified, the presence of a second QTL was investigated by using the two QTL model. In subsequent steps, most significant QTL were fitted as background effects to detect additional QTL. The inclusion of previously detected QTL to the standard model decreases the error variance and thereby increases the statistical power to detect QTL. This procedure was repeated until no additional significant QTL was detected. Empirically derived significance thresholds were estimated by random QTL scans with 1000 permutations of the data. The $95 \%$-confidence interval of a single QTL was estimated using a parametric bootstrap analysis with 1000 iteration.

For fine mapping study, paired-end reads were trimmed using TRIMMOMATIC with standard settings using the TruSeq3-PE adapter sequences provided by Illumina for adapter trimming. Reads were aligned against the Gallus gallus 4 reference genome (Ensembl 78) using BWA (version 0.7.10), after which GATK and PICARD tools were applied to perform base quality score recalibration, indel realignment, duplicate removal, and SNP and INDEL discovery across all samples simultaneously using the GATK HAPLOTYPECALLER with variant quality score recalibration according to GATK Best Practices recommendations. Pairwise tests of LD were performed for used markers in every generation using the SHESIS software platform (http://analysis.biox.cn/myAnalysis.php). We used the LME4 package in $\mathrm{R}$ (version 3.1.3) to perform the association analysis between genotypes and growth or body composition traits (see Lyu et al. 2017 for detail).

\section{Findings and Discussion}

In chicken, linkage and physical maps, as well as sequence data and bioinformatics tools are available that makes whole-genome scans for QTL and QTG feasible. Our data confirmed that the phenotypic differences between the crossed lines were of polygenic nature. Because of the high correlation among different analysed traits, we expected to identify QTL that had effects on more than one trait (Figure 1). Some loci had specific effect in the early or late growth performance.

This project confirms that most genetic effects contributing to our growth-related traits act additively (Chicken QTLdb release 34; http://www.animalgenome.org/cgi-

bin/QTLdb/GG/index). Most alleles inherited from the $\mathrm{NHI}$ line were associated with higher performance for identified loci. Thus, our data clearly provide evidence that many loci across the genome have responded to the selection procedure for high meat yield in the line NHI.

We mapped several genomic regions on 22 chromosomes affecting 24 phenotypes (Figure 1). 
Linkage analysis provided evidence for highly significant QTL on GGA1, 2, 4, 5, 7, 10, 12, 15, 26 and 27. The highest QTL effects accounting for 4.6 to 40.2 $\%$ of the phenotypic $\mathrm{F}_{2}$ variance were found on the distal region of GGA4 between 42.1 and $88.4 \mathrm{Mb}(F \geq 11.2)$. The QTL allele of the high weight NHI line had positive additive effects on body weight, body weight gain, muscle mass and carcass traits $(14.0 \mathrm{~g} \leq \mathrm{a} \leq 141.8 \mathrm{~g})$, and had negative additive effect on adipose tissue mass $(-5.3 \mathrm{~g})$. We suggest a transgressive action of the obesity allele only if it is not in the genetic background of the line WL77 (Nassar et al. 2013). Transgressive QTL alleles (high-fat alleles inherited from the lean line WL77) can occur in a mapping population because of no or limited selection for these traits, drift, pleiotropic effects of the QTL allele, linkage to genes affecting other traits that are under selection, as result of interaction between genes, or as modifier alleles (Abasht et al. 2006). The change in the direction of effect can occur when crossing populations with different genetic backgrounds (Nassar et al. 2013). Transgressive allele effects for the same chromosomal region were also reported by Nadaf et al. (2009).

Using body weight as a covariate in the analysis of body composition traits provided evidence for genes in the GGA4 QTL region affecting adipose tissue mass independently of body weight. The QTL effect size differed between sexes and depended on the direction of cross. The results confirmed known QTL and identified new QTL effects on GGA5, 7, 8, 9, 10, 11, 12, 13, 14, 15, 16, 18, 24, 26, 27 and 28. For intramuscular fat content, a suggestive QTL was located on GGA14. The detection of multiple traits in the same genomic region could be caused on the molecular level either by several genes in a narrow chromosomal region affecting these traits or by a single mutation that affects all traits in a pleiotropic manner.

The size of the GGA4 region is large $(26.9 \mathrm{Mb})$, where hundreds of genes (QTG) reside that could potentially affect the growth traits (Nassar et al. 2015). This promising genomic region has been repeatedly mapped for growth and body composition traits in several crosses worldwide (Chicken QTLdb release 34; http://www.animalgenome.org/cgi-

bin/QTLdb/GG/index), in which traits were analysed at younger or older ages, shows that these genomic loci affect the traits during a long developmental period. In crosses between different breeds, e.g. White Leghorn (layer) with either broiler (meat-type chicken) (Sewalem et al. 2002; Carlborg et al. 2003, 2004; Schreiweis et al. 2006; Podisi et al. 2011), Rhode Island Red (TuiskulaHaavisto et al. 2002; Sasaki et al. 2004), red junglefowl (Kerje et al. 2003; Le Rouzic et al. 2008), or Oh-Shamo (native Japanese breed) (Tsudzuki et al. 2007), as well as in crosses layer lines (Zhou et al. 2006; Ambo et al. 2009) the same region on GGA4 affected growth. In addition, the effect of this QTL on growth performance has been noticed too in Egyptian chicken breeds or lines; Fayoumi (Zhou et al. 2006a, b), Golden Montazah (Abdel Alal et al. 2016), line CE1 that has been selected for 6-week body weight for 15 generations and line CE2 as the genetic control (Higazy et al. 2017). Therefore, our findings together with published results obtained in other crosses could be relevant for Egyptian chicken breeds.

Because the locus on chromosome 4 has been identified in many crosses between egg and meat-type chickens, either layer or broiler lines could have a joined phylogenetic origin, as suggested by the phylogenetic tree analysis of sequence variances between domestic chickens and their subsequent specialization into broiler and layer (Rubin et al. 2010). As the magnitudes of the genetic effects reported for the different mapping populations vary, it can be assumed that very likely more than two alleles occur in the different breeds. Therefore, the high QTL effects identified in our population and others can contribute to identify QTG (functional candidate genes) and their causative polymorphism(s). 


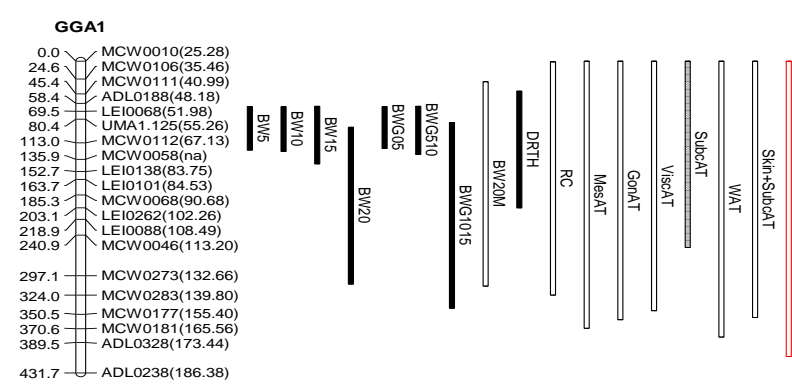

GGA6

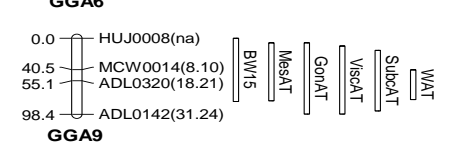

$0.0-$ Rosoo78(1.91)

$74.4-$ MCW0135(13.66)

${ }_{120.9-1}^{102.9-A D L 0136(n a)}$ MCW0149(23.77)

GGA12

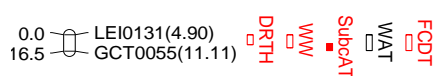

GGA15

0.0
8.6

GGA24 GGA26

29.6 LEI0155(4.11)

GGA2

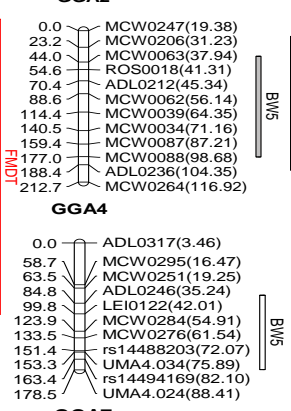

GGA7

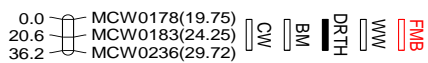

\section{GGA10}

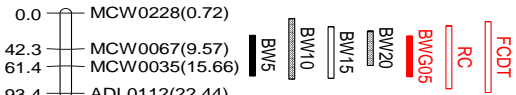

GGA13

$0.0-M C W 0340(1.07)$

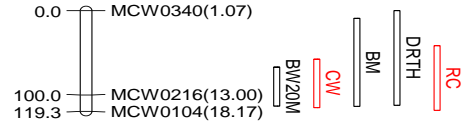

GGA16

$0.0-$ LEIO258(0.10)

GGA27

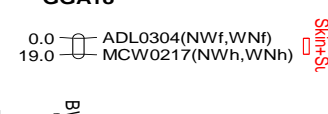

GGA3

.0 MCW0261(0.80

43.6 - ADL0177(6.84)

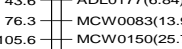

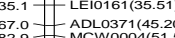

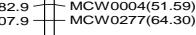

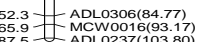

0.0
3.6

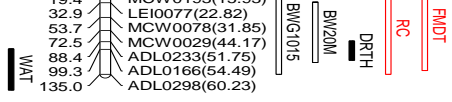

GGA8

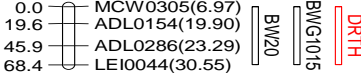

GGA11

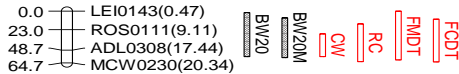

GGA14

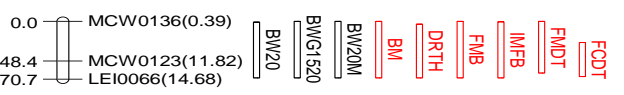

GGA20

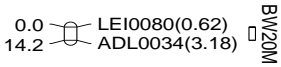

GGA28

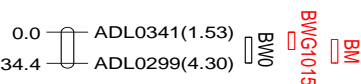

Figure 1 Marker map and position of QTL identified in $\mathrm{F}_{2}$ populations of reciprocal crosses between NHI and WL77 for meat production traits [Nassar et al. 2012 , 2013 , 2015]. On the left side of every chromosome, marker positions are given in cM, on the right side marker names are given with positions in Mb in brackets. Bars on the right side of every chromosome represent genome-wide suggestive (open), significant (striped), and highly significant (filled) QTL with 95\% confidence intervals. Red color represents QTL effects that have not been reported before. GGA = Gallus gallus autosome; BW0, BW5, BW10, BW15, BW20 = Body weight at hatch, 5, 10, 15, and 20 weeks of age, respectively; BWG05, BWG510, BWG1015, BWG1520 = Body weight gain as difference between two subsequent body weight measurements, e.g. between hatch and 5, 5 and 10, 10 and 15; and 15 and 20 weeks, respectively. $\mathbf{B W 2 0 M}=\mathrm{Body}$ weight at 20 weeks in males; $\mathbf{B M}=$ Breast muscle weight; CW = Carcass weight; DRTH = Drumsticks and thighs weight; FCDT = Fat content in drumsticks and thighs; FMB = Fat mass in breast muscles; FMDT = Fat mass in drumsticks and thighs; GonAT = Gonadal adipose tissue; IMFB = Intramuscular fat in breast muscles; MesAT = Mesenteric adipose tissue; $\mathbf{R C}=$ Residual carcass weight Skin + subcAT $=$ Skin + subcutaneous adipose tissues; SubcAT $=$ Subcutaneous neck adipose tissue; ViscAT = Visceral adipose tissues; WAT = Total white adipose tissues; $\mathbf{W W}=$ Wings weight. 
For many linkage analyses obvious functional candidate genes are not immediately evident. Comparative sequencing of all genes within the confidence intervals of identified QTL is usually not achievable. Therefore, fine mapping is necessary to physically reduce the numbers of QTG. Albeit QTL identified in our study were localized in same chromosomal regions in other crosses, every cross descending from a different genetic recourse population can contribute to reduce the target chromosomal region and haplotypes most likely harbouring the quantitative trait nucleotides (QTN) (Li et al. 2005; Brockmann et al. 2009; Tortereau et al. 2010; Rückert and Bennewitz 2010).

The support confidence interval of the GGA4 QTL region between 61.5 and $88.4 \mathrm{Mb}$ in the chicken genome harbours 292 genes (Ensemble release 77) that could potentially affect the analysed traits. Therefore, we performed fine mapping to physically reduce the chromosomal interval and the number of potential candidate genes. An advanced intercross line has been established from the initial $\mathrm{F}_{2}$ mapping population, in which we used generations $F_{10}$ to $F_{12}$. The linkage disequilibrium between markers/genes in this population decreases from generation to generation (Besnier et al. 2011). Nine SNP markers within the QTL confidence interval region were selected to perform an association analysis with several growth traits from hatch to 20 weeks and body composition traits at 20 weeks. The confidence interval of the QTL has been reduced from 26.9 to $3.4 \mathrm{Mb}$. Within the fine mapped region, markers $r s 14490774, r s 314961352$ and $r s 318175270$ were in full linkage disequilibrium and showed the strongest effect on growth and muscle mass (LOD $\geq 4$ ). This reduced region contains 30 genes, compared to 292 genes in the original region (Nassar et al. 2015; Lyu et al. 2017). Chicken $60 \mathrm{~K}$ and $600 \mathrm{~K}$ SNP chips combined with full genome sequencing of the parental lines were used to call mutations in the reduced region. In the narrowed-down region 489 sequence variants were detected. Variant effect prediction showed that $47 \%$ of the 489 variants were intergenic. The most deleterious variants are a missense variant in ADGRA3 and a frameshift deletion in the functional unknown gene ENSGALG00000014401. In addition, five synonymous variants were discovered in the genes PPARGC1A, ADGRA3, PACRGL, SLIT2 and FAM184B (Lyu et al. 2016, 2017).

\section{Conclusions}

The large differences in growth and body composition traits between the parental lines permitted the detection of genomic regions and genes affecting the analysed phenotypes in the reciprocal $F_{2}$ crosses and AIL animals. Our study together with published results obtained in other crosses confirmed the importance of the distal
GGA4 region for chicken growth. The confidence interval and the number of potential genes could be reduced 8- and 10- fold respectively. Variant calling in the reduced QTL region provided more knowledge on this QTL. Further research will focus on functional effects of mutant genes. The final identification of gene(s) and their causative mutation(s) contributes to our understanding of the complex inheritance pattern of growth regulation, muscle development and fat deposition in chicken. Such information could be relevant for local chicken breeds as well as for commercial practice for improving growth quality and quantity using molecular selection in breeding scheme.

\section{References}

Abasht B., Dekkers J.C.M. and Lamont S.J. (2006). Review of quantitative trait loci identified in the chicken. Poultry Science 85: 2079-2096.

Abdel Alal M.H., Khalil M.H., Iraqi M.M. and ElMoghazy Gihan M. (2016). Quantitative trait loci affecting growth performance in $\mathrm{F}_{2}$ intercross between Golden Montazah and White Leghorn chickens. $3^{\text {rd }}$ International Conference on Biotechnology Applications in Agriculture (ICBAA), Benha University, Moshtohor and Sharm El-Sheikh, 5-9 April 2016, Egypt.

Abiola O., Angel J.M., Avner P., Bachmanov A.A., Belknap J.K., Bennett B., Blankenhorn E.P., Blizard D.A., Bolivar V., Brockmann G.A., Buck K.J., Bureau J.F., Casley W.L., Chesler E.J., Cheverud J.M., Churchill G.A., Cook M., Crabbe J.C., Crusio W.E., Darvasi A., de Haan G., Dermant P., Doerge R.W., Elliot R.W., Farber C.R., Flaherty L., Flint J., Gershenfeld H., Gibson J.P., Gu J., Gu W., Himmelbauer H., Hitzemann R., Hsu H.C., Hunter K., Iraqi F.F., Jansen R.C., Johnson T.E., Jones B.C., Kempermann G., Lammert F., Lu L., Manly K.F., Matthews D.B., Medrano J.F., Mehrabian M., Mittlemann G., Mock B.A., Mogil J.S., Montagutelli X., Morahan G., Mountz J.D., Nagase H., Nowakowski R.S., O'Hara B.F., Osadchuk A.V., Paigen B., Palmer A.A., Peirce J.L., Pomp D., Rosemann M., Rosen G.D., Schalkwyk L.C., Seltzer Z., Settle S., Shimomura K., Shou S., Sikela J.M., Siracusa L.D., Spearow J.L., Teuscher C., Threadgill D.W., Toth L.A., Toye A.A., Vadasz C., Van Zant G., Wakeland E., Williams R.W., Zhang H.G. and Zou F. (2003). The nature and identification of quantitative trait loci: a community's view. Nature Reviews Genetics 4: 911-916.

Alfonso L. and Haley C.S. (1998). Power of different $\mathrm{F}_{2}$ schemes for QTL detection in livestock. Animal Science 66: 1-8. 
Ambo M., Moura A.S.A.M.T., Ledur M.C., Pinto L.F.B., Baron E.E., Ruy D.C., Nones K., Campos R.L.R., Boschiero C., Burt D.W. and Coutinho L.L. (2009). Quantitative trait loci for performance traits in a broiler x layer cross. Animal Genetics 40: 200-208.

Andersson L. (2001). Genetic dissection of phenotypic diversity in farm animals. Nature Reviews Genetics 2: $130-138$.

Besnier F., Wahlberg P., Rönnegård L., Ek W., Andersson L., Siegel P.B. and Carlborg O. (2011). Fine mapping and replication of QTL in outbred chicken advanced intercross lines. Genetics Selection Evolution 43: 3-12.

Brockmann G.A., Tsaih S.W., Neuschl C., Churchill G.A. and Li R.H. (2009). Genetic factors contributing to obesity and body weight can act through mechanisms affecting muscle weight, fat weight, or both. Physiological Genomics 36: 114126.

Campos R.L., Nones K., Ledur M.C., Moura A.S., Pinto L.F., Ambo M., Boschiero C., Ruy D.C., Baron E.E., Ninov K., Altenhofen C.A., Silva R.A., Rosario M.F., Burt D.W. and Coutinho L.L. (2009). Quantitative trait loci associated with fatness in a broiler-layer cross. Animal Genetics 40: 729-736.

Carlborg O., Hocking P.M., Burt D.W. and Haley C.S. (2004). Simultaneous mapping of epistatic QTL in chickens reveals clusters of QTL pairs with similar genetic effects on growth. Genetical Research 83: 197-209.

Carlborg O., Kerje S., Schutz K., Jacobsson L., Jensen P. and Andersson L. (2003). A global search reveals epistatic interaction between QTL for early growth in the chicken. Genome Research 13: 413421.

Goraga Z., M. Nassar, G-P. Schramm and G.A. Brockmann (2010). Phenotypic characterization of chicken inbred lines that differ extremely in growth, body composition, and egg production traits. Archives Animal Breeding 3: 337-349.

Goraga Z.S., M.K. Nassar and G.A. Brockmann (2012). Quantitative trait loci segregating in crosses between New Hampshire and White Leghorn chicken lines: I. Egg production traits. Animal Genetics 43: $183-189$.

Higazy D., El-Kashef A. and El-Gendy, E. (2017). Detection of microsatellite loci linked to QTL for growth in warm-region chickens under natural prolonged heating conditions. Proceeding of the 2017 International poultry scientific forum, pp: 15-16.

Ikeobi C.O.N., Woolliams J.A., Morrice D.R., Law A., Windsor D., Burt D.W. and Hocking P.M. (2004). Quantitative trait loci for meat yield and muscle distribution in a broiler layer cross. Livestock Production Science 87: 143-1451.
Jacobsson L., Park H.B., Wahlberg P., Fredriksson R., Perez-Enciso M., Siegel P.B. and Andersson L. (2005). Many QTLs with minor additive effects are associated with a large difference in growth between two selection lines in chickens. Genetical Research 86: 115-125.

Jennen D., Vereijken A., Bovenhuis H., Crooijmans R., Veenendaal A., van der Poel J. and Groenen M. (2004). Detection and localization of quantitative trait loci affecting fatness in broilers. Poultry Science 83: 295-301.

Kerje S., Carlborg O., Jacobsson L., Schutz K., Hartmann C., Jensen P. and Andersson L. (2003). The twofold difference in adult size between the red jungle fowl and White Leghorn chickens is largely explained by a limited number of QTLs. Animal Genetics 34: 264-274.

Khalil M.H., Abdel Alal M.H., Iraqi M.M. and ElMoghazy Gihan M. (2016). QTL and chromosomal mapping for growth and egg performance in chickens: Applications and emphasis of results in Egypt. $3^{\text {rd }}$ International Conference on Biotechnology Applications in Agriculture (ICBAA), Benha University, Moshtohor and Sharm El-Sheikh, 5-9 April 2016 , Egypt.

Le Rouzic A., Alvarez-Castro J.M. and Carlborg O. (2008). Dissection of the genetic architecture of body weight in chicken reveals the impact of epistasis on domestication traits. Genetics 179: 1591-1599.

Li R., Lyons M.A., Wittenburg H., Paigen B. and Churchill G.A. (2005). Combining data from multiple inbred line crosses improves the power and resolution of quantitative trait loci mapping. Genetics 169: 1699-1709.

Lyu S., D. Arends, M.K. Nassar and G.A. Brockmann (2016). Fine mapping of a distal chromosome 4 QTL affecting growth and muscle mass in a chicken advanced intercross line. Journal of Animal Science 94 (7supplement4), 132-133.

Lyu S., D. Arends, M.K. Nassar and G.A. Brockmann (2017). Fine mapping of a distal chromosome 4 QTL affecting growth and muscle mass in a chicken advanced intercross line. Animal Genetics 48: 295302.

Nadaf J., Pitel F., Gilbert H., Duclos M.J., Vignoles F., Beaumont C., Vignal A., Porter T.E.,Cogburn L.A., Aggrey S.E., Simon J. and Le Bihan-Duval E. (2009). QTL for several metabolic traits map to loci controlling growth and body composition in an $\mathrm{F}_{2}$ intercross between high- and low-growth chicken lines. Physiological Genomics 38: 241-249.

Nassar M.K. and G.A. Brockmann (2011). Genetic factors contributing to fat deposition in chicken. Poultry Science 90(E-Suppl. 1) 5. 
Nassar M.K. and G.A. Brockmann (2013). Mapping QTL affecting growth performance in chicken. Poultry Science 92(E-Suppl. 1) 104.

Nassar M.K., Z.S. Goraga and G.A. Brockmann (2012). Quantitative trait loci segregating in crosses between New Hampshire and White Leghorn chicken lines: II. Muscle weight and carcass composition. Animal Genetics 43: 739-745.

Nassar M.K., Z.S. Goraga and G.A. Brockmann (2013). Quantitative trait loci segregating in crosses between New Hampshire and White Leghorn chicken lines: III. Fat deposition and intramuscular fat content. Animal Genetics 44: 62-68.

Nassar M.K., Z.S. Goraga and G.A. Brockmann (2015). Quantitative trait loci segregating in crosses between New Hampshire and White Leghorn chicken lines: IV. Growth performance. Animal Genetics 46: 441-446.

Park H.-B., Jacobsson L., Wahlberg P., Siegel P.B. and Andersson L. (2006). QTL analysis of body composition and metabolic traits in an intercross between chicken lines divergently selected for growth. Physiological Genomics 25: 216-223.

Podisi B.K., Knott S.A., Dunn I.C., Law A.S., Burt D.W. and Hocking P.M. (2011). Overlap of quantitative trait loci for early growth rate, and for body weight and age at onset of sexual maturity in chickens. Reproduction 141: 381-389.

Rubin C.J., Zody M.C., Eriksson J., Meadows J.R., Sherwood E., Webster M.T., Jiang L., Ingman M., Sharpe T., Ka S., Hallbook F., Besnier F., Carlborg O., Bed'hom B., Tixier-Boichard M., Jensen P., Siegel P., Lindblad-Toh K. and Andersson L. (2010). Whole-genome resequencing reveals loci under selection during chicken domestication. Nature 464: 587-591.

Rückert C. and Bennewitz J. (2010). Joint QTL analysis of three connected $\mathrm{F}_{2}$-crosses in pigs. Genetics Selection Evolution 42: 40.

Sasaki O., Odawara S., Takahashi H., Nirasawa K., Oyamada Y., Yamamoto R., Ishii K., Nagamine Y., Takeda H., Kobayashi E. and Furukawa T. (2004). Genetic mapping of quantitative trait loci affecting body weight, egg character and egg production in $\mathrm{F}_{2}$ intercross chickens. Animal Genetics 35: 188-194.

Schreiweis M.A., Hester P.Y., Settar P. and Moody D.E. (2006). Identification of quantitative trait loci associated with egg quality, egg production, and body weight in an $\mathrm{F}_{2}$ resource population of chickens. Animal Genetics 37: 106-112.

Schreiweis M.A., Hester P.Y. and Moody D.E. (2005). Identification of quantitative trait loci associated with bone traits and body weight in an $F_{2}$ resource population of chickens. Genetics Selection Evolution 37: 677-698.
Sewalem A., Morrice D., Law A., Windsor D., Haley C., Ikeobi C., Burt D. and Hocking P. (2002). Mapping of quantitative trait loci for body weight at three, six, and nine weeks of age in a broiler layer cross. Poultry Science 81: 1775-1781.

Tortereau F., Gilbert H., Heuven H.C., Bidanel J.P., Groenen M.A. and Riquet J. (2010). Combining two Meishan $F_{2}$ crosses improves the detection of QTL on pig chromosomes 2, 4 and 6. Genetics Selection Evolution 42: 42.

Tsudzuki M., Onitsuka S., Akiyama R., Iwamizu M., Goto N., Nishibori M., Takahashi H. and Ishikawa A. (2007). Identification of quantitative trait loci affecting shank length, body weight and carcass weight from the Japanese cockfighting chicken breed, Oh-Shamo (Japanese Large Game). Cytogenetic and Genome Research 117: 288-295.

Tuiskula-Haavisto M., Honkatukia M., Vilkki J., de Koning D.J., Schulman N.F. and Maki- Tanila A. (2002). Mapping of quantitative trait loci affecting quality and production traits in egg layers. Poultry Science 81: 919-927.

Zhou H., Deeb N., Evock-Clover C.M., Ashwell C.M. and Lamont S.J. (2006a). Genome-wide linkage analysis to identify chromosomal regions affecting phenotypic traits in the chicken. I. Growth and average daily gain. Poultry Science 85: 1700-1711.

Zhou H., Deeb N., Evock-Clover C.M., Ashwell C.M. and Lamont S.J. (2006b). Genome-wide linkage analysis to identify chromosomal regions affecting phenotypic traits in the chicken. II. Body composition. Poultry Science 85: 1712-1721. 
